\title{
TURBULENCE-FLAME INTERACTIONS IN TYPE Ia SUPERNOVAE
}

\author{
A. J. Aspden, ${ }^{1}$ J. B. Bell, ${ }^{1}$ M. S. Day, ${ }^{1}$ S. E. Woosley, ${ }^{2}$ and M. Zingale ${ }^{3}$ \\ Received 2008 May 27; accepted 2008 August 10
}

\begin{abstract}
The large range of time and length scales involved in Type Ia supernovae (SNe Ia) requires the use of flame models. As a prelude to exploring various options for flame models, we consider in this paper high-resolution, three-dimensional simulations of the small-scale dynamics of nuclear flames in the supernova environment in which the details of the flame structure are fully resolved. The range of densities examined, $(1-8) \times 10^{7} \mathrm{~g} \mathrm{~cm}^{-3}$, spans the transition from the laminar flamelet regime to the distributed burning regime where small-scale turbulence disrupts the flame. The use of a low Mach number algorithm facilitates the accurate resolution of the thermal structure of the flame and the inviscid turbulent kinetic energy cascade, while implicitly incorporating kinetic energy dissipation at the grid-scale cutoff. For an assumed background of isotropic Kolmogorov turbulence with an energy characteristic of SNe Ia, we find a transition density between 1 and $3 \times 10^{7} \mathrm{~g} \mathrm{~cm}^{-3}$, where the nature of the burning changes qualitatively. By $1 \times 10^{7} \mathrm{~g} \mathrm{~cm}^{-3}$, energy diffusion by conduction and radiation is exceeded, on the flame scale, by turbulent advection. As a result, the effective Lewis number approaches unity. That is, the flame resembles a laminar flame but is turbulently broadened with an effective diffusion coefficient, $D_{T} \sim u^{\prime} l$, where $u^{\prime}$ is the turbulent intensity and $l$ is the integral scale. For the larger integral scales characteristic of a real supernova, the flame structure is predicted to become complex and unsteady. Implications for a possible transition to detonation are discussed.
\end{abstract}

Subject headings: conduction - hydrodynamics - methods: numerical — nuclear reactions, nucleosynthesis, abundances - supernovae: general - turbulence - white dwarfs

Online material: color figure

\section{INTRODUCTION}

The complex small-scale dynamics of turbulent thermonuclear flames are essential to understanding SNe Ia. Since the range of length scales is so large (star size $\sim 10^{8} \mathrm{~cm}$; Kolmogorov scale and flame thickness $<1 \mathrm{~mm}$ ), full star calculations must use a subgrid model to describe the burning on the unresolved scales. Turbulent flame models (Niemeyer \& Hillebrandt 1995) that move the flame at the turbulent velocity have been successful at producing explosions (Röpke \& Hillebrandt 2005) with pure deflagrations. Another popular flame model (Khokhlov 1991) moves the flame at the speed dictated by the Rayleigh-Taylor instability and has also produced successful explosions (Gamezo et al. 2003), although the authors argue that a more realistic supernova is produced when a detonation ensues late in the explosion (Gamezo et al. 2005). These two flame models can produce large differences in the flame speeds at small scales, which will have a large effect on the outcome of full-star simulations. Validation of these models against resolved flame calculations is needed to resolve this discrepancy.

As the flame propagates outward from the center of the star, it encounters lower densities, the speed decreases, and the flame thickens. A critical length scale in turbulent combustion is the Gibson scale - the length scale at which the laminar flame speed equals the turbulent velocity (see, e.g., Peters 2000),

$$
l_{\mathrm{G}}=l\left(\frac{s_{L}}{u^{\prime}}\right)^{3}
$$

\footnotetext{
1 Lawrence Berkeley National Laboratory, 1 Cyclotron Road, MS 50A-1148, Berkeley, CA 94720.

${ }^{2}$ Department of Astronomy and Astrophysics, University of California at Santa Cruz, Santa Cruz, CA 95064.

${ }^{3}$ Department of Physics and Astronomy, Stony Brook University, Stony Brook, NY 11794.
}

where $s_{L}$ is the laminar flame speed, and $u^{\prime}$ and $l$ are velocity and integral length scales characterizing the turbulence, and we have assumed Kolmogorov scaling. At a density of $\sim 10^{7} \mathrm{~g} \mathrm{~cm}^{-3}$, the flame becomes thick enough that turbulent eddies can disrupt its structure before they burn away (Niemeyer \& Woosley 1997), that is, the flame thickness is larger than the Gibson scale. At this point, the burning fundamentally changes character and the flame is said to be in the distributed burning regime (see Peters 2000 for some discussion).

Here we look at the interaction of the flame and turbulence on the scale of the flame, with the aim of validating turbulent flame models and better understanding the transition to distributed burning. Previous studies of flames interacting with turbulence have focused on flames interacting with vortical flow (see the twodimensional simulations presented in Röpke et al. 2004) or statistical methods using one-dimensional turbulence (Lisewski et al. 2000a, 2000b). An open question is whether in the distributed burning regime a mixed region of partially burned fuel and ash can grow large enough that it can ignite a detonation (Niemeyer \& Woosley 1997; Khokhlov et al. 1997; Niemeyer 1999). Detonations have gained renewed interest lately as a means to burn the carbon/oxygen fuel left behind near the center of the white dwarf in pure deflagration models (Gamezo et al. 2005). However, it is unclear whether detonations can traverse the pockets of partially burned fuel (Golombek \& Niemeyer 2005). Recent results also suggest that the size of the region of partially burned fuel needed to initiate a detonation is larger than previously believed (Dursi \& Timmes 2006). Pan et al. (2008) discusses the role of turbulent intermittency on the conditions needed for transition to detonation.

Previously, we showed the transition to the distributed burning regime in two dimensions (Bell et al. 2004b), where the Rayleigh-Taylor (RT) instability, growing on scales smaller than the flame thickness itself, is responsible for the transition. It has 


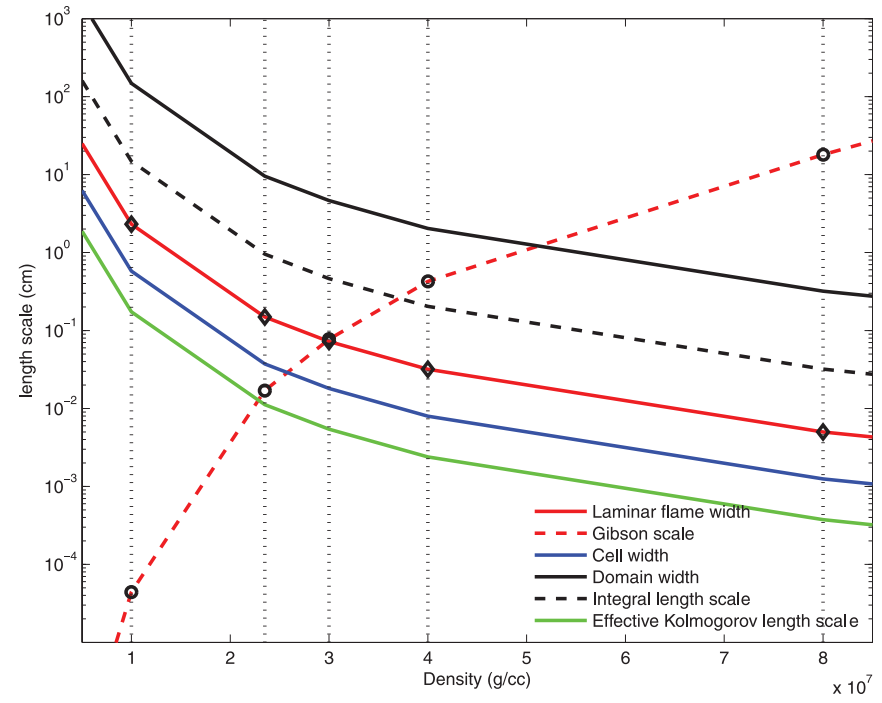

FIG. 1.-Comparison of the variation of length scales at different fuel densities. The solid red line shows how the laminar flame width increases with decreasing density. The dashed red line shows how the Gibson scale $\left(l_{\mathrm{G}}\right)$ decreases with density (Kolmogorov scaling appropriate for a star has been assumed; see eq. [1]). The flame thickness and Gibson scale are approximately equal at a density of $\rho_{7} \approx 3$. This marks the transition between the flamelet and distributed burning regimes. The cell width is shown by the solid blue line; the resolution of the turbulent simulations was chosen such that $l_{L}=4 \Delta x$. The available computational resources limit the size of calculation, so the domain width in each case was $L=256 \Delta x$ and is shown by the solid black line. The forcing term used to maintain the turbulence imposes an integral length scale that is approximately a tenth of the domain width and is shown by the dashed black line. Aspden et al. (2008) demonstrated that the effective Kolmogorov length scale using this numerical scheme is approximately $0.28 \Delta x$ and is shown by the solid green line. The vertical dashed lines denote the densities of the five turbulent simulations.

been suggested (Niemeyer \& Kerstein 1997) that turbulence generated by RT instability in SNe Ia should obey BolgianoObukhov (BO) statistics - formulated from considering a potential energy cascade. However, recently, it was shown that BO scaling only applies in two dimensions (Chertkov 2003; Zingale et al. 2005a). Three-dimensional RT-unstable flame calculations have shown that turbulence is indeed Kolmogorov in nature and becomes isotropic on the small scales (Zingale et al. 2005b; Cabot $\&$ Cook 2006). As first discussed in Niemeyer \& Kerstein (1997), BO scaling leads to a lower transition to distributed burning and therefore makes a detonation transition more difficult. Therefore, although our two-dimensional results led us to conclude that a deflagration to detonation transition was unlikely (Bell et al. 2004b), a three-dimensional study is warranted.

Figure 1 shows how the relevant length scales vary with fuel density. In particular, the two red curves compare the laminar flame width and the Gibson scale. The laminar flame widths have been calculated from simulated one-dimensional profiles. The Gibson scale is evaluated assuming a turbulent intensity $u^{*}$ of $10^{7} \mathrm{~cm} \mathrm{~s}^{-1}$ on a length scale $L^{*}$ of $10^{6} \mathrm{~cm}$, as in Niemeyer $\&$ Woosley (1997). We see that the transition to distributed burning, assuming Kolmogorov turbulence, should occur around $3 \times 10^{7} \mathrm{~g} \mathrm{~cm}^{-3}$.

In this paper, we present a three-dimensional study of turbulent thermonuclear flames that explores a range of conditions from the flamelet regime to the distributed burning regime. Specifically, we use a flame sheet embedded in a maintained turbulent velocity field. Of particular interest is the response of the flames to wrinkling by the turbulence, and the scaling of the turbulent flame speed with the turbulent intensity. Thermal diffusion is orders of magnitude greater than species diffusion (the Lewis number is large; Timmes \& Woosley 1992) and has a significant effect on the turbulence-flame interactions. Attention is then focused on the distributed burning regime, and potential implications for a transition to detonation are discussed.

\section{SIMULATION DESCRIPTION}

We use a low Mach number hydrodynamics code, adapted to the study of thermonuclear flames, as described in Bell et al. (2004a). The advantage of this method is that sound waves are filtered out analytically, so the time step is set by the bulk fluid velocity and not the sound speed. This is an enormous efficiency gain for low-speed flames. The input physics used in the present simulations is largely unchanged, with the exception of the addition of Coulomb screening, taken from the Kepler code (Weaver et al. 1978), to the ${ }^{12} \mathrm{C}\left({ }^{12} \mathrm{C}, \gamma\right){ }^{24} \mathrm{Mg}$ reaction rate. This yields a small enhancement to the flame speed and is included for completeness. The conductivities are those reported in Timmes (2000), and the equation of state is the Helmholtz free-energy based general stellar EOS described in Timmes \& Swesty (2000). We note that we do not utilize the Coulomb corrections to the electron gas in the general EOS, as these are expected to be minor in the conditions considered.

The nonoscillatory finite-volume scheme employed here permits the use of implicit large eddy simulation (ILES). This technique captures the inviscid cascade of kinetic energy through the inertial range, while the numerical error acts in a way that emulates the physical effects of the dynamics at the grid scale, without the expense of resolving the entire dissipation subrange. The approach was introduced by Boris et al. (1992) and has since been used by many authors (e.g., Youngs 1991; Porter et al. 1992; Grinstein \& Fureby 2004; Margolin et al. 2006). An overview of the technique can be found in Grinstein et al. (2007). Aspden et al. (2008) presented a detailed study of the technique using the present numerical scheme, including a characterization that allowed for an effective viscosity to be derived. Thermal diffusion plays a significant role in the flame dynamics and so is explicitly included in the model, whereas species diffusion is significantly smaller and so is not explicitly included.

The turbulent velocity field was maintained using a forcing term similar to that used in the study of Aspden et al. (2008). Specifically, a forcing term was included in the momentum equations consisting of a superposition of long-wavelength Fourier modes with random amplitudes and phases. The forcing term is scaled by density so that the forcing is somewhat reduced in the ash. This approach provides a way to embed the flame in a zero-mean turbulent background, mimicking the much larger inertial range that these flames would experience in an SN Ia, without the need to resolve the large-scale convective motions that drive the turbulent energy cascade. Figure 2 shows an example kinetic energy wavenumber spectrum taken from Aspden et al. (2008) at a resolution comparable to that used here; the dashed line denotes a minus five-thirds decay and is illustrative of the turbulence found in the present study. Aspden et al. (2008) demonstrated that the effective Kolmogorov length scale is approximately $0.28 \Delta x$, and the integral length scale is approximately a tenth of the domain width.

Figure 3 shows the simulation setup. The simulations were initialized with carbon fuel in the lower part of the domain and magnesium ash in the upper, resulting in a downward propagating flame. A high-aspect ratio domain was used to allow the flame sufficient space to propagate. Periodic boundary conditions were prescribed laterally, along with a free-slip base, and outflow at the upper boundary.

Five simulations were run to investigate the turbulence-flame interactions in different burning regimes. The five cases will be 


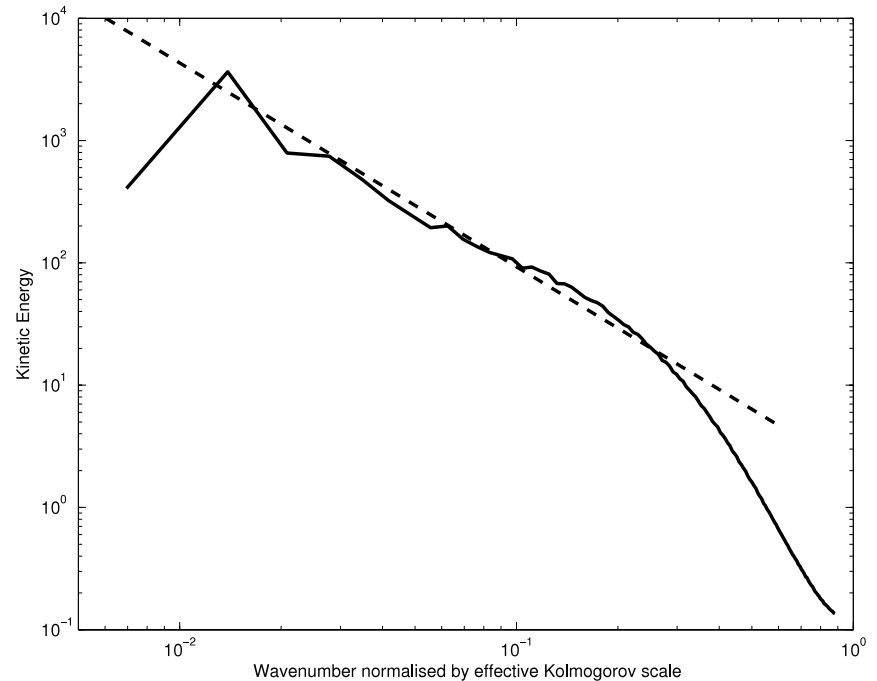

FIG. 2.-Kinetic energy wavenumber spectrum from a homogeneous isotropic turbulence simulation at $256^{3}$, taken from Aspden et al. (2008). The same forcing term used to maintain the turbulence is used in the present flame calculations. This figure demonstrates the range of scales captured by the ILES method for turbulent flow simulations at the resolution used in the present study. The dashed black line denotes the minus five-thirds decay expected in an inertial range. [See the electronic edition of the Journal for a color version of this figure.]

referred to as cases (a) through (e). The study was designed so that $l_{\mathrm{G}} / l_{L}$ varied across a number of orders of magnitude, from approximately $4 \times 10^{3}$ in case (a) corresponding to the flamelet regime, to $2 \times 10^{-5}$ in case (e) corresponding to the distributed burning regime. The different burning regimes were achieved by varying the density of the carbon fuel from $8 \times 10^{7}$ for case (a)

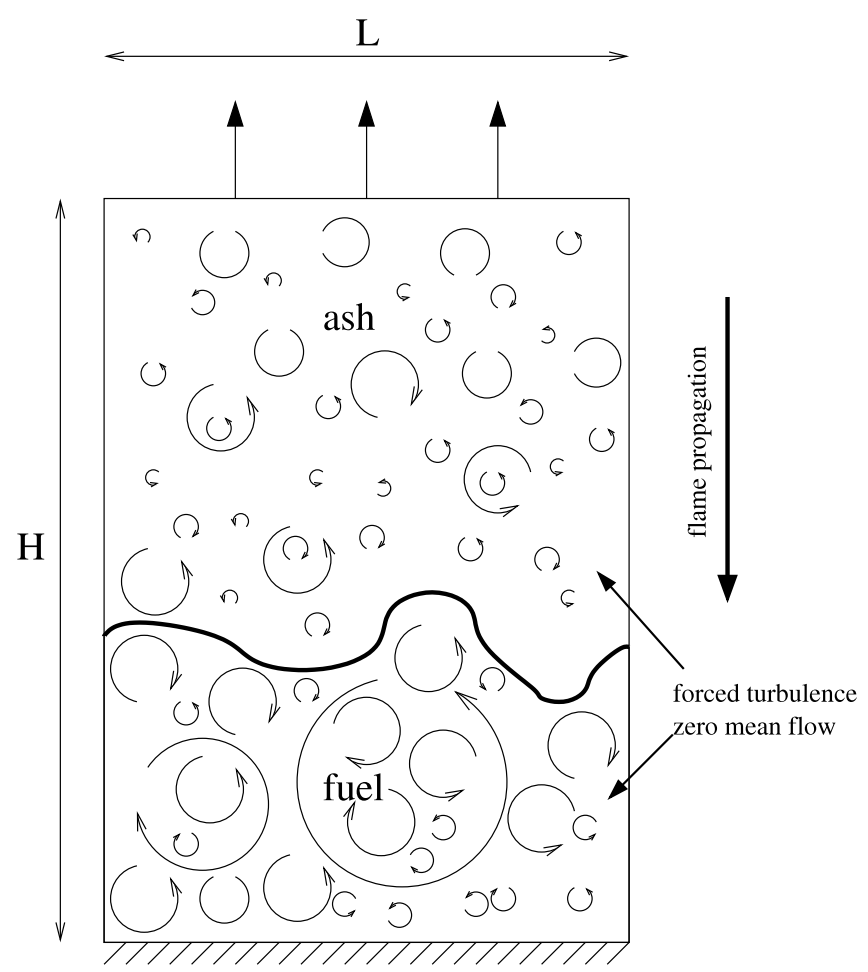

FIG. 3.-Diagram of the simulation setup (shown in two dimensions for clarity). The domain is initialized with a turbulent flow, and a flame is introduced into the domain, oriented so that the flame propagates toward the lower boundary. The turbulence is maintained by adding a forcing term to the momentum equations. The top and bottom boundaries are outflow and solid wall boundaries, respectively. The side boundaries are periodic.

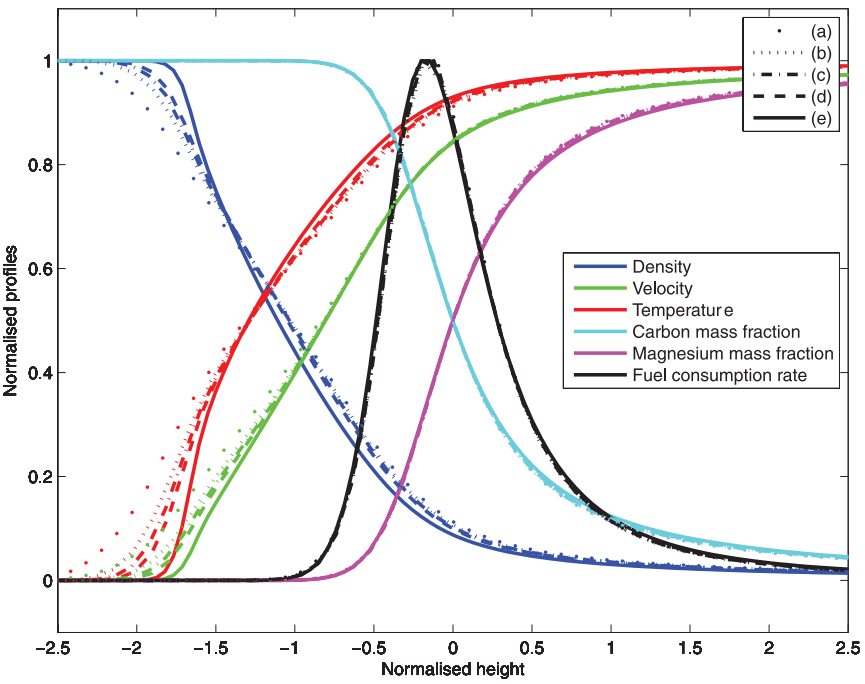

FIG. 4.- Normalized laminar flame profiles. Each color denotes a different quantity, as indicated by the legend. The line style corresponds to the five different cases; the dotted line is case (a) through to the solid line for case (e). The length scales have been normalized by the laminar flame width, defined to be the carbon width $l_{L}=\left(\Delta X_{\mathrm{C}}\right) / \max \left|\nabla X_{\mathrm{C}}\right|$, where $X_{\mathrm{C}}$ is the carbon mass fraction. Each quantity has been normalized by the minimum and maximum values attained (see Table 2$), \bar{q}=\left(q(z)-q_{\min }\right) /\left(q_{\max }-q_{\min }\right)$. Note the velocity is given in the frame of reference of the flame.

down to $1 \times 10^{7}$ in case (e). Case (a) corresponds to near the center of a supernova, has a thin laminar flame, and relatively low turbulence levels. Case (e) corresponds to the conditions near the edge of a supernova, has a thicker laminar flame, and relatively more intense turbulence. The derivation of the turbulence conditions will be described below.

For each case, a flat laminar flame simulation was first run to steady state to establish a laminar flame width and speed. The laminar flame width was taken to be the carbon width, specifically $l_{L}=\left(\Delta X_{\mathrm{C}}\right) / \max \left|\nabla X_{\mathrm{C}}\right|$, where $X_{\mathrm{C}}$ is the carbon mass fraction. The variation of laminar flame thickness with fuel density is shown by the solid red curve in Figure 1. The laminar flame solution also provides initial flame conditions for the turbulent simulations. Figure 4 shows normalized laminar profiles for the five cases. The length scale has been normalized by the carbon width, and each quantity has been normalized by the minimum and maximum value across the domain, i.e., $\bar{q}=\left(q-q_{\min }\right) /$ $\left(q_{\max }-q_{\min }\right)$ for a generic quantity $q$. Properties of the laminar flames are summarized in Table 1 .

Based on the resolution studies presented in Bell et al. (2004a, $2004 b$ ), the resolution for the turbulent flame simulations was chosen to have four cells across the laminar flame width. Specifically, the carbon profile from each laminar flame simulation was measured, $l_{L}$, and $\Delta x$ set to $l_{L} / 4$, which is shown by the solid blue line in Figure 1, and, following Aspden et al. (2008), the effective Kolmogorov length scale is shown by the solid green line. This choice of cell width corresponds to many more cells across the entire flame; at the resolution of the turbulent flame simulations to follow, the spatial extent shown in Figure 4 would be spanned by 20 computational cells. Since it is expected that the turbulence will thicken the flame, this choice of resolution is believed to be sufficient to resolve the reaction zone of the turbulent flame.

Computational expense restricts the domain to a cross section of 256 cells, and so, since $\Delta x$ is known, the domain width follows, and the forcing term determines the integral length scale. The domain width and integral length scales are shown in Figure 1 
TABLE 1

Laminar Flame Properties

\begin{tabular}{|c|c|c|c|c|c|}
\hline \multirow[b]{2}{*}{ Parameter } & \multicolumn{5}{|c|}{ CASE } \\
\hline & (a) & (b) & (c) & (d) & (e) \\
\hline 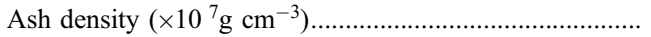 & 5.74 & 2.64 & 1.91 & 1.43 & 0.52 \\
\hline 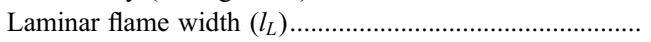 & $5.00 \times 10^{-3}$ & $3.19 \times 10^{-2}$ & $7.24 \times 10^{-2}$ & $1.49 \times 10^{-1}$ & 2.31 \\
\hline 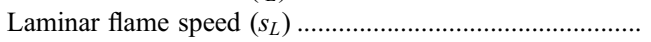 & $2.62 \times 10^{5}$ & $7.52 \times 10^{4}$ & $4.26 \times 10^{4}$ & $2.57 \times 10^{4}$ & $3.54 \times 10^{3}$ \\
\hline Laminar peak fuel consumption rate $\left(\mathrm{g} \mathrm{cm}^{-3} \mathrm{~s}^{-1}\right) \ldots \ldots \ldots . .$. & $2.10 \times 10^{15}$ & $4.82 \times 10^{13}$ & $8.96 \times 10^{12}$ & $2.04 \times 10^{12}$ & $7.61 \times 10^{9}$ \\
\hline Laminar velocity change & $1.03 \times 10^{5}$ & $3.85 \times 10^{4}$ & $2.45 \times 10^{4}$ & $1.63 \times 10^{4}$ & $3.24 \times 10^{3}$ \\
\hline 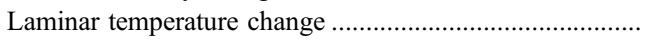 & $4.20 \times 10^{9}$ & $3.60 \times 10^{9}$ & $3.37 \times 10^{9}$ & $3.18 \times 10^{9}$ & $2.57 \times 10^{9}$ \\
\hline
\end{tabular}

by the solid and dashed black lines, respectively. It should be emphasized that the integral length scale and effective Kolmogorov length scales are restricted by computational expense and are not reflective of the values found in a supernova.

Turbulence characteristics can be estimated using a simple representation shear generation by the Rayleigh-Taylor instability. On the large scale of the supernova, the flame will be unstable with a typical feature size of $L^{*} \sim 10^{6} \mathrm{~cm}$. The velocity on this scale can be estimated using the Sharp-Wheeler model (Sharp 1984),

$$
u^{*}\left(L^{*}\right) \sim \frac{1}{2} \sqrt{\operatorname{At} g L^{*}} .
$$

For an Atwood number, At $\sim 0.5$, and gravitational acceleration, $g \sim 10^{9} \mathrm{~cm} \mathrm{~s}^{-2}$, one has $u^{*}\left(L^{*}\right) \sim 10^{7} \mathrm{~cm} \mathrm{~s}^{-1}$, in agreement with the numbers used in Niemeyer \& Woosley (1997). More detailed simulations by Röpke (2007) in 3D find a distribution of turbulent intensities peaking at $10^{7} \mathrm{~cm} \mathrm{~s}^{-1}$, but with a high-velocity tail extending out to $10^{8} \mathrm{~cm} \mathrm{~s}^{-1}$. For the current simulations, the turbulent kinetic energy released by the large-scale RT motions is assumed to cascade down to the flame scale following a Kolmogorov spectrum, as

$$
u^{\prime}(l)=u^{*}\left(L^{*}\right)\left(\frac{l}{L^{*}}\right)^{1 / 3} ;
$$

therefore, given the integral length scale, $l$, the turbulent velocity $u^{\prime}(l)$ can be computed. Throughout this paper, $u^{*}=10^{7} \mathrm{~cm} \mathrm{~s}^{-1}$ and $L^{*}=10^{6} \mathrm{~cm}$ have been assumed.

In cases (a)-(d), the aspect ratio of the domain was $4: 1$ and the flame placed one domain width from the upper boundary. In case (e) an aspect ratio of 8:1 was required to accommodate the flame in the distributed burning regime, and the flame was initially placed at the midpoint of the domain. In each case, adaptive mesh refinement (AMR) was used to reduce the computational expense. A base grid with 128 cells across was used, with one level of AMR with refinement factor 2 concentrated around the flame sheet. This gave an effective resolution of $256 \times 256 \times 1024$ for cases (a) -(d) and $256 \times 256 \times 2048$ for case (e). Experiments with additional resolution confirmed the adequacy of this choice. It was also ensured that the use of AMR had no detrimental effect on the turbulence. Specifically, the energy containing scales were well captured by the base grid (see Aspden et al. [2008] for a study of maintained homogeneous isotropic turbulence using the same approach), and there was adequate refinement ahead of the flame to ensure that the cascade of energy to smaller scales in the refined region (which have a short turn over time) could occur before these scales could interact with the flame. Table 2 summarizes the properties of the turbulence simulations.

\section{RESULTS}

In each case, the flow undergoes an initial transition, as the turbulence wrinkles and thickens the flame, until a quasi-steady state is established. Figure 5 shows instantaneous vertical slices of fuel consumption rate (left panel) and temperature (right panel) for each of the five cases once the quasi-steady state has been reached. In each case, the values have been normalized by the corresponding laminar values, with the exception of case (e), where the local fuel consumption rate is much lower and so has been normalized by a fifth of the laminar value for contrast. By construction, the length scales are normalized by the laminar flame width. Half of the domain is shown for cases (a)-(c) and case (e), and the entire domain is shown for case (d).

Case (a) presents smooth and even burning and is perturbed very little by the background turbulence. The temperature profile

TABLE 2

\begin{tabular}{|c|c|c|c|c|c|}
\hline \multirow[b]{2}{*}{ Parameter } & \multicolumn{5}{|c|}{ CASE } \\
\hline & (a) & (b) & (c) & (d) & (e) \\
\hline Fuel density $\left(\times 10^{7} \mathrm{~g} \mathrm{~cm}^{-3}\right) \ldots \ldots \ldots$ & 8 & 4 & 3 & 2.35 & 1 \\
\hline 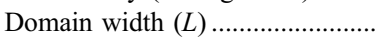 & 0.32 & 2.0 & 4.6 & 9.5 & 150 \\
\hline 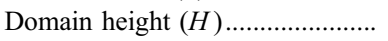 & 1.28 & 8.0 & 18.4 & 38.0 & 1200 \\
\hline Integral length scale $(l) \ldots \ldots \ldots \ldots \ldots$ & 0.032 & 0.2 & 0.46 & 0.95 & 15 \\
\hline 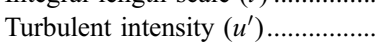 & $3.17 \times 10^{4}$ & $5.85 \times 10^{4}$ & $7.72 \times 10^{4}$ & $9.83 \times 10^{4}$ & $2.47 \times 10^{5}$ \\
\hline Gibson scale $\left(l_{\mathrm{G}}\right) \ldots \ldots$ & $1.8 \times 10^{1}$ & $4.3 \times 10^{-1}$ & $7.7 \times 10^{-2}$ & $1.7 \times 10^{-2}$ & $4.4 \times 10^{-5}$ \\
\hline Karlovitz number $\sqrt{l_{L} / l_{\mathrm{G}}} \ldots \ldots \ldots \ldots$ & $1.67 \times 10^{-2}$ & 0.274 & 0.968 & 2.96 & $2.28 \times 10^{2}$ \\
\hline
\end{tabular}

Simulation Pproperties. 

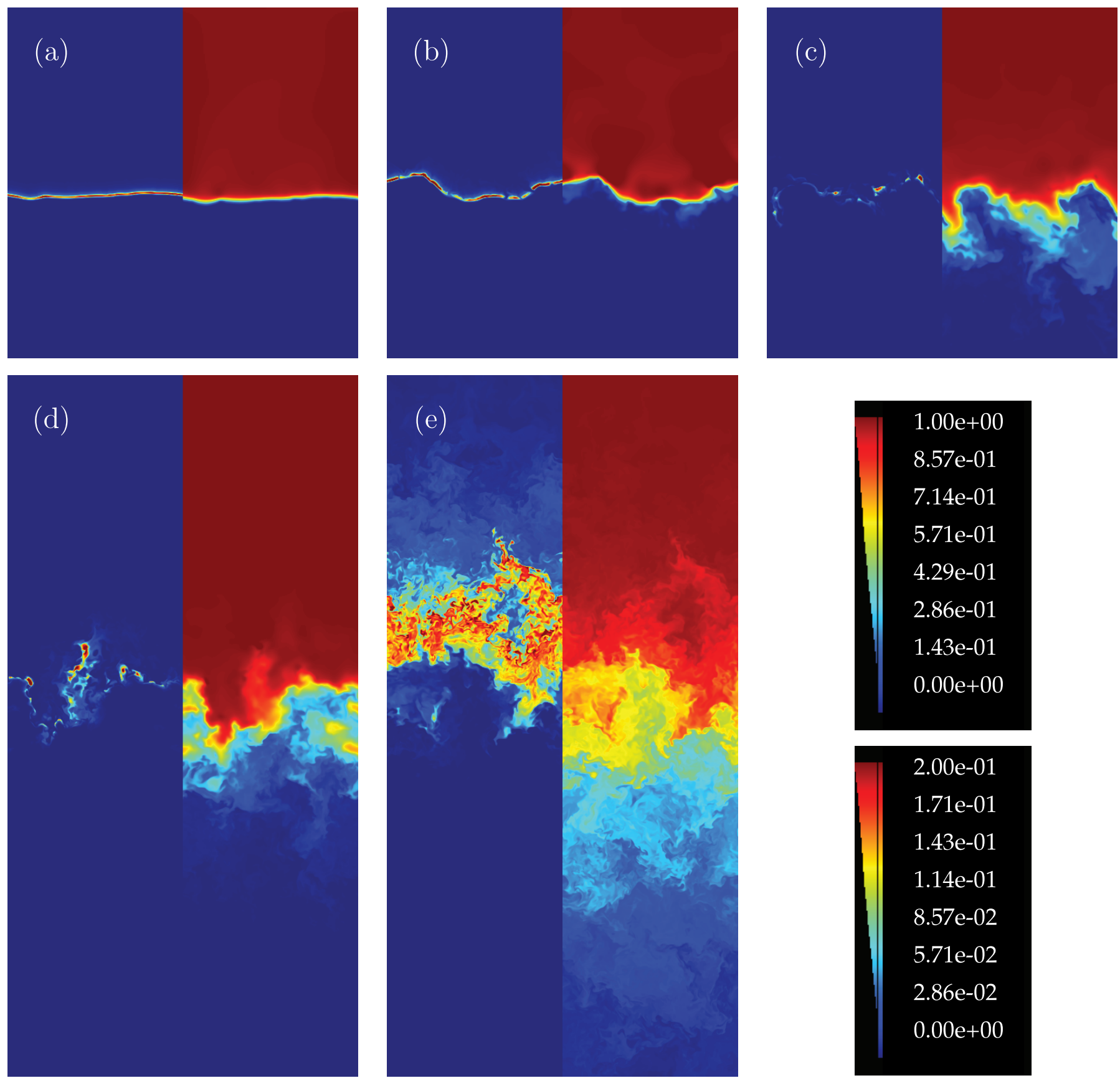

FIg. 5.- Instantaneous vertical slices through each flame. In each case, the left-hand panel shows the fuel consumption rate, normalized by the corresponding laminar value [except for case (e), which was normalized by one-fifth of the laminar value because it burns much less intensely than the laminar flame], and the right-hand panel shows the temperature field, again normalized by the laminar value (refer to Table 2 for the values). The top legend shows the range for each normalized value except fuel consumption rate in case (e), which is shown by the lower legend.

remains sharp. In case (b), the flame surface has been deformed by the turbulence. Both regions of enhanced burning and regions of decreased burning are observed, and appear to be correlated with the curvature of the flame sheet. Specifically, enhanced burning appears to occur where the center of curvature is within the fuel and reduced burning where the center of curvature is in the ash. The temperature field presents regions that are sharp and regions that appear to be more diffuse. Again, this appears to be correlated with curvature, with the more diffusive regions occurring where the center of curvature is in the products.

In cases (c) and (d), as $l_{\mathrm{G}} / l_{L}$ decreases further, the background turbulence becomes increasingly influential; the temperature field becomes more mixed, and the deformation of the flame surface increases. The burning appears to occur in small high-intensity pockets, punctuated by regions of local extinction.

In case (e), a dramatically different burning mode is observed. The temperature-mixed region and the burning region are much broader. The burning appears to be much less intense (recall the image has been normalized by a fifth of the laminar value for contrast) and is restricted to the high-temperature end of the mixing zone. There is no well-defined flame surface, but a broad flame brush. Interestingly, there appears to be some residual lowlevel burning well above the main burning region, suggestive of incomplete burning in the main flame zone.

These observations are further reinforced by three-dimensional renderings of the fuel consumption rate, shown in Figure 6, which 

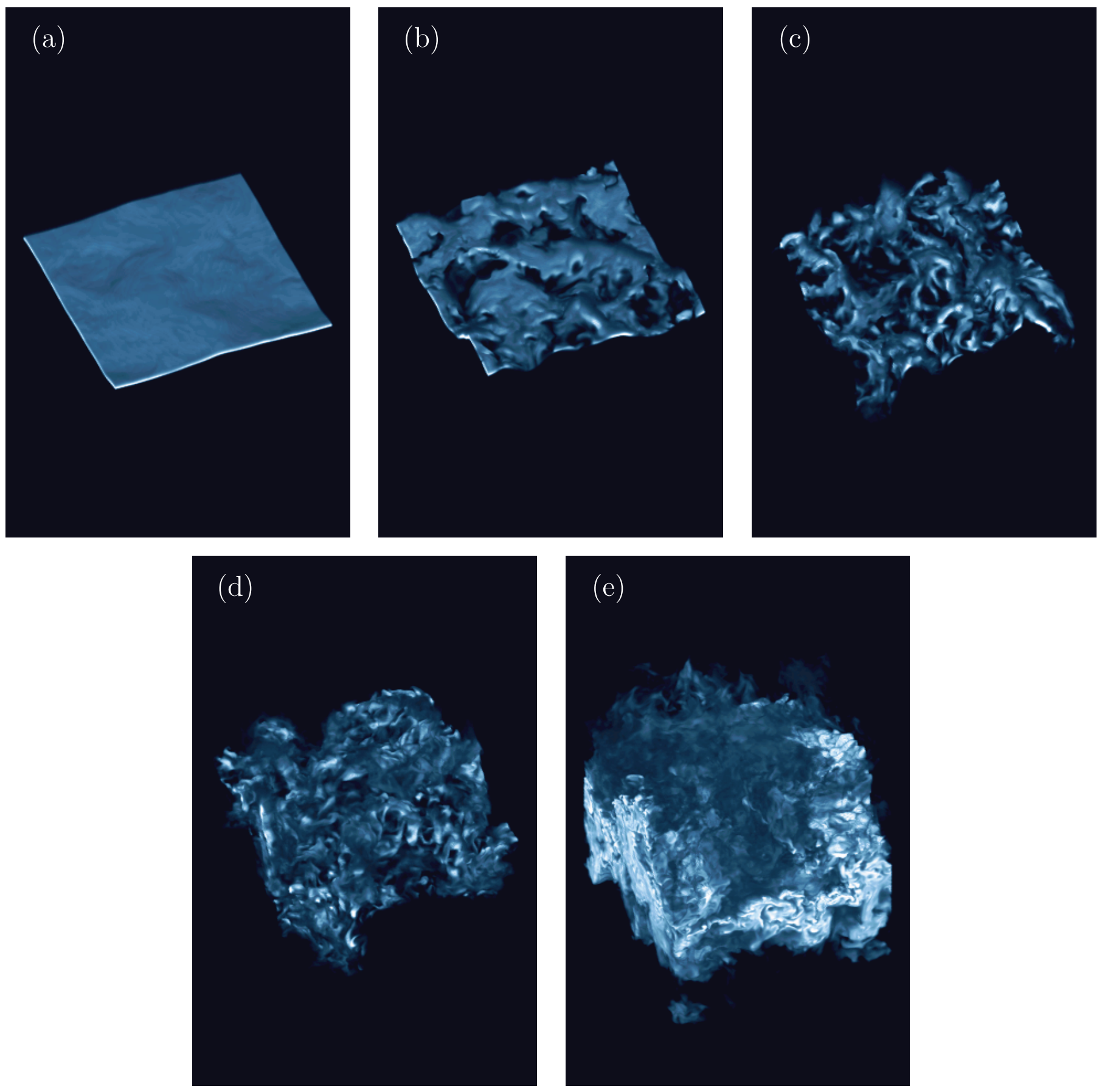

FIG. 6.-Three-dimensional instantaneous fuel consumption rate in each of the five cases, rendered such that the burning intensity is linearly related to the opacity of the image. In the flamelet regime, the flame is coherent and flat. As the relative turbulence increases, the flame becomes increasingly disrupted, which leads to regions of enhanced burning and regions of local extinction. Finally, in the distributed burning regime in case (e), the character of the burning changes dramatically; the flame is greatly broadened and burns much less intensely (recall that the normalization is by a fifth of the laminar value), but the overall fuel consumption rate is enhanced.

elucidates the three-dimensional structure of the flames. The images have been scaled in the same way as the corresponding slices. Case (a) burns as a coherent connected flame sheet, but as the relative turbulence level increases in cases (b)-(d), the flame sheet becomes increasingly disrupted and presents regions of local extinction. Finally, in case (e), in the distributed burning regime, the behavior is completely different with a flame brush that is much broader but burns less intensely than the laminar flame.

The curvature effects observed and the resulting burning rates are a consequence of the thermodiffusively stable nature of the flames; the Lewis number is high - thermal diffusion is much greater than species diffusion. Where the background turbulence elevates part of the flame surface, resulting in negative curvature, there is a focusing of heat by diffusion. Consequently, as the reaction rate is extremely sensitive to temperature, the fuel burns quickly, and the flame is flattened. Conversely, where the turbulence pushes the flame downward, creating positive curvature, temperature diffusion leads to a defocusing of heat, and the burning rate decreases. Again, this tends to flatten the flame. Curvature effects will be explored further below. 


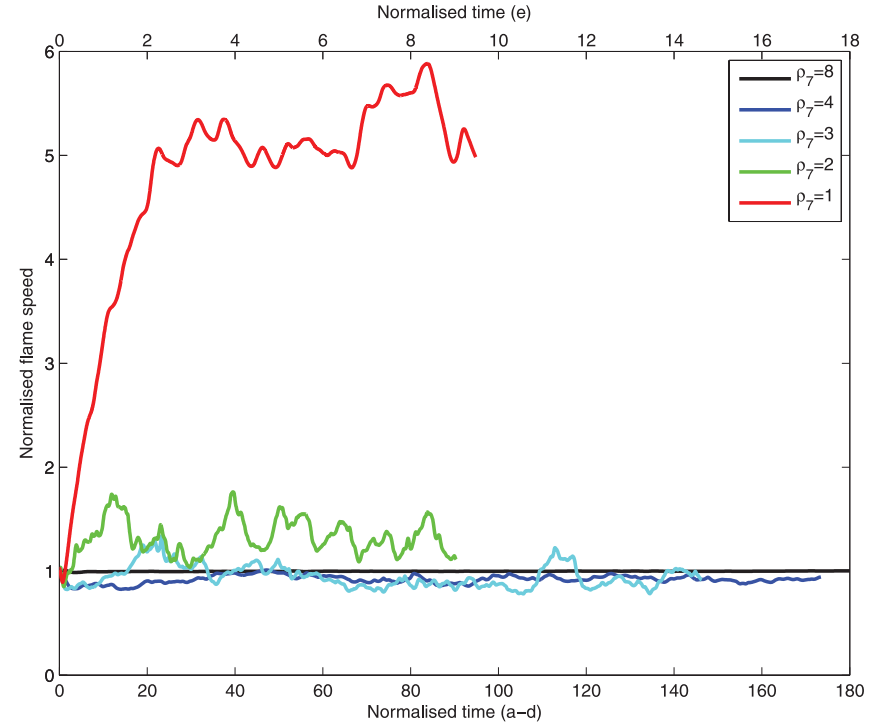

FIG. 7.- Normalized turbulent flame speeds. The timescale has been normalized by the laminar burning time, i.e., the time taken to burn one laminar flame width, specifically $l_{L} / s_{L}$. Cases (a)-(d) use the lower timescale; case (e), which has a significantly different evolution, uses the upper timescale. The turbulent flame speed has been normalized by the laminar flame speed.

The instantaneous global turbulent flame speed normalized by the laminar flame speeds are shown in Figure 7; the timescale is normalized by the laminar burning time - the time it takes the laminar flame to burn one flame width. Note the different time axis for case (e). Case (a) burns almost exactly at the laminar flame speed. Curiously, cases (b) and (c) actually burn more slowly overall than their laminar counterparts, and case (d) is only $\sim 30 \%$ faster. However, case (e) burns between approximately 5 and 6 times the laminar flame speed.

To investigate the effects of curvature, it is useful to be able to define a flame surface. There are a number of ways to do this, but it has been found that using isosurfaces of temperature or fuel mass fraction is a practical way that avoids the difficulties associated with the local extinction in these particular flames. We choose values for the isosurfaces based on the temperature or fuel mass fraction values at the peak local fuel consumption rate in the laminar flame. The isosurface was then located by using a standard "marching cubes" algorithm and optimized using the "QSlim" algorithm (see Garland 1999). Since the temperature field is more diffusive than the fuel, the resulting isosurface was found to be much smoother for temperature than the carbon, particularly where the isosurface was within the fuel itself. Furthermore, because of the higher levels of turbulence in cases (d) and (e), the isosurfaces were found to be too contorted to be useful.

Since the fuel consumption occurs over a finite width and is not localized to the flame surface, a local consumption-based flame speed was evaluated by integrating the fuel consumption rate through the surface. This involved constructing a set of integral curves along the gradient vector of the progress variable through each point on the isosurface, ensuring that the curves extended well beyond the region where the fuel consumption rate decreases to zero. (We note that for this construction, we orient the normal to point into the ash. With this orientation, the curvature $\kappa$ is negative when the center of curvature is in the fuel.) These integral curves provided bounding edges of prism-shaped subvolumes that effectively cover the reaction zone. The consumption-based flame propagation speed was then computed over each subvolume of the reaction zone by integrating the computed fuel con-

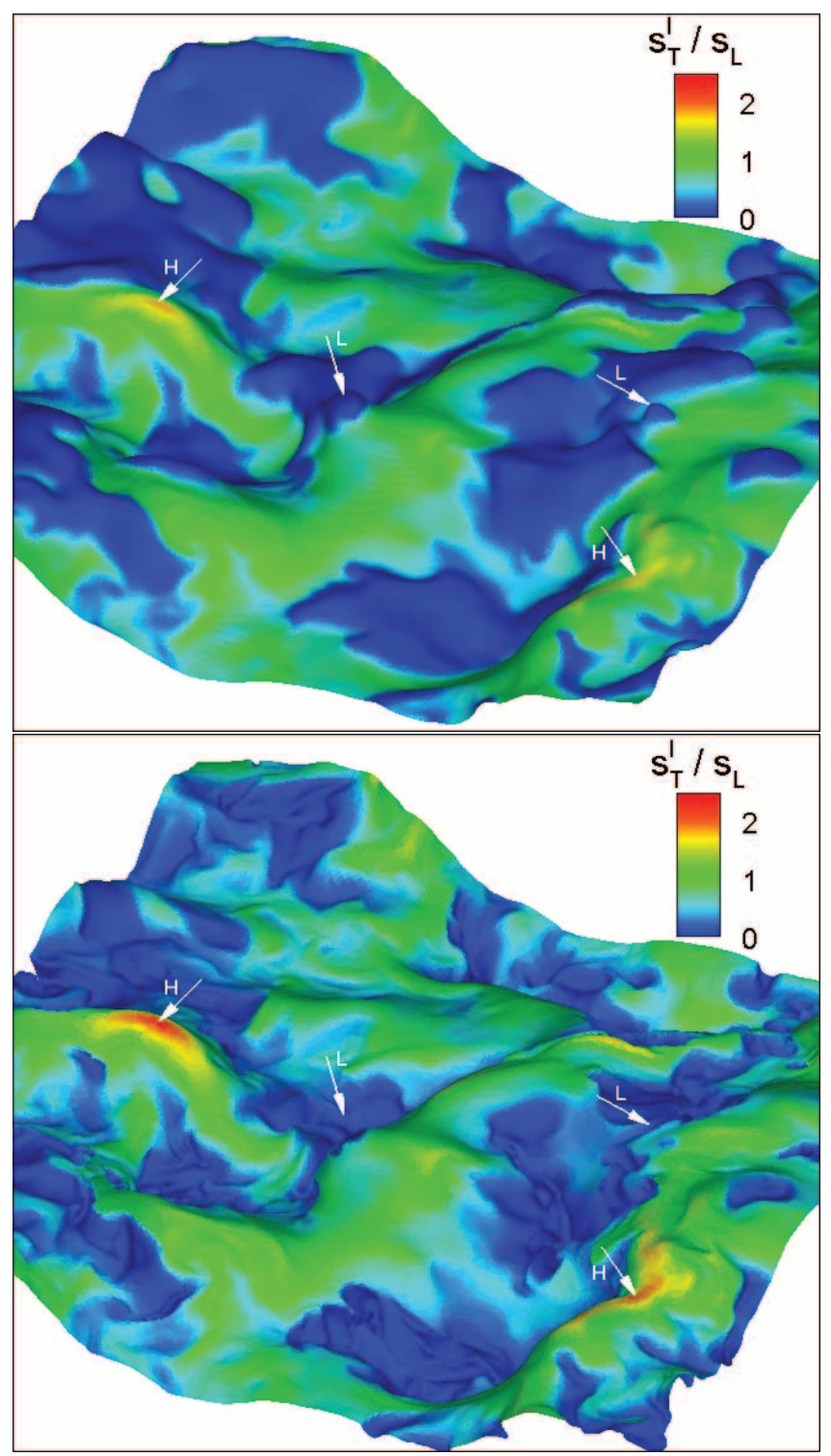

FIG. 8.-Comparison of isosurfaces based on temperature (top) and carbon mass fraction (bottom) for case (b). The surfaces are colored by the locally integrated fuel consumption rate (see the text and eq. [4]). The arrows labeled " $H$ " and "L" highlight regions of high and low fuel consumption rate, respectively, both of which are correlated with negative curvature in the temperature isosurface. However, in the carbon isosurface, the surface is much lower, indicating that although the fluid is at a sufficient temperature to burn, the fuel is absent.

sumption rate over the subvolume. The local consumption-based flame speed was defined as

$$
s_{T}^{l}=\frac{1}{\rho_{0} X_{F, 0} A} \int_{\Omega} \rho \dot{\omega}_{F} d \Omega,
$$

where $\rho_{0} X_{F, 0}$ is the initial fuel density, $\Omega$ is the subvolume, $\rho \dot{\omega}_{F}$ is the local fuel consumption rate, and $A$ is the area of intersection of the flame with $\Omega$. Defining the local speed in this way has the property that the global burning speed is the integral of $s_{T}^{l}$ over the isosurface. For additional details about construction of the elements $\Omega$, see Bell et al. (2006) and Day et al. (2008).

Figure 8 compares instantaneous temperature and carbon isosurfaces for case (b) and is colored by the integrated fuel consumption rate, $s_{T}^{l}$. The correlation between burning rate and curvature observed in Figure 5 is more apparent here. Specifically, the regions 

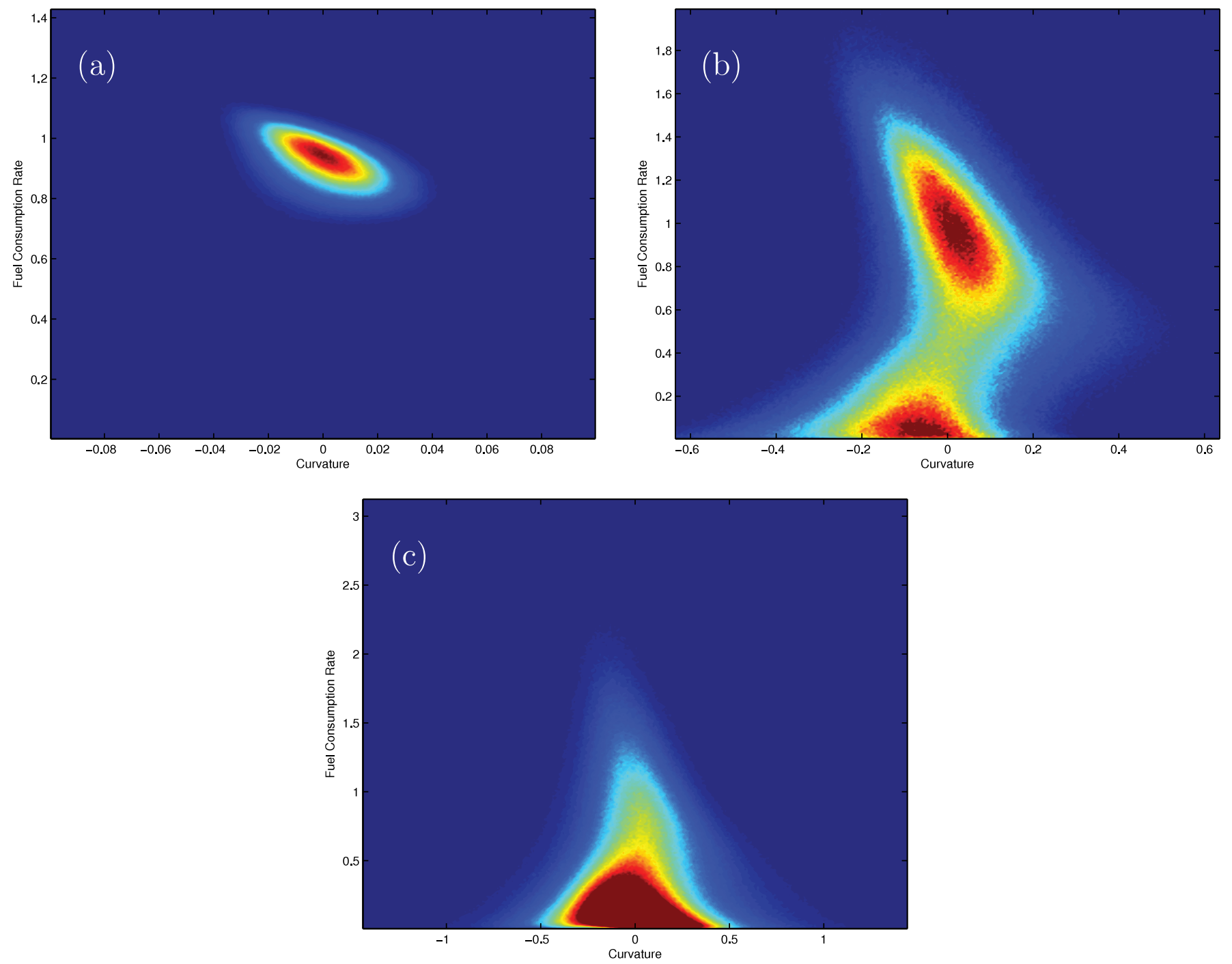

Fig. 9.- Joint probability density functions of the locally integrated fuel consumption rate $\left(s_{T}^{l}\right)$ normalized by the laminar flame speed $\left(s_{L}\right)$ against curvature of the temperature isosurface $\left(\kappa_{T}\right)$ normalized by the laminar flame width $\left(l_{L}\right)$; (a) $\rho_{7}=8$, (b) $\rho_{7}=4$, (c) $\rho_{7}=3$. The (negative) correlation is clear, specifically that the fuel consumption rate increases with negative curvature. For case (b), the regions of negative curvature labeled "L" in Fig. 8, with low fuel consumption rate, explain the bimodal PDF here.

of high fuel consumption rate are strongly correlated with regions of negative curvature, i.e., where the centers of curvature are within the fuel. Two examples are highlighted by arrows labeled "H." This correlation appears to differ slightly between the two isosurfaces. On the temperature surface, there are regions of negative curvature where the burning is very low; two examples are shown by arrows labeled "L." However, the carbon surface does not appear to pass through these regions; the labels are in the same place, but the carbon surface is much lower. The temperature and carbon mass fraction have become decorrelated. Regions exists around the peak burning temperature where there is no fuel, and so burning cannot occur. This is a direct result of the competition between mixing by turbulence and thermal diffusion.

Figure 9 shows joint probability density functions (JPDFs) of curvature (normalized by the laminar flame width) and local integrated fuel consumption rate (normalized by the laminar value), based on the temperature isosurface, ensemble-averaged over a number of time points after the flames have reached a quasisteady state. The (negative) correlation is clear and quantifies the relationship between the curvature and local flame speed ob- served above. It is also evident that there is a significant change in burning across the three cases. Case (a) shows that the majority of the fuel consumption occurs around the laminar rate. Case (b) demonstrates that the flame is bimodal, in the sense that there are regions that are burning, which are negatively correlated with curvature, but there are also regions of low burning or local extinction, which occur with curvature of both signs. This suggests that where the flame is burning, it is burning close to the laminar value (with low probability variability), but because there are also regions of local extinction, the overall burning rate per unit area is lower than the laminar value. Finally, in case (c), there is no preferred rate of burning, a slight negative correlation with curvature, and significant regions of local extinction.

A common approach used for determining a model flame speed involves assuming that the flame is burning locally at the laminar flame speed, and that the global burning rate is equal to the laminar flame speed times the area of the flame. Figure 10 shows the normalized burning rate per unit area for cases (a)-(c). If the flame-modeling approach described above was appropriate for these flames, this measure would be close to unity. This is indeed seen to be the case for case (a), as the flame is burning in a very 


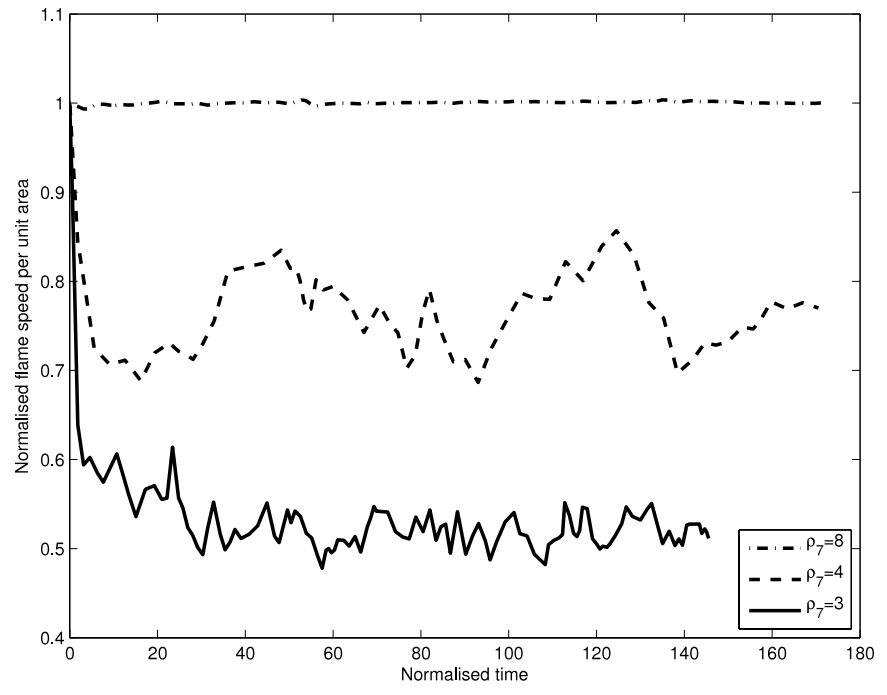

FIG. 10.-Normalized turbulent flame speed per unit area. The turbulent flame speed has been normalized by the laminar flame speed, and the area has been taken to be the area of the temperature isosurface. The timescale has been normalized by the laminar burning time $\left(l_{L} / s_{L}\right)$. This demonstrates that a Markstein correction is required to construct a flame model that captures the curvature dependence of the flame speed.

similar way to the laminar flame. However, it is too simplistic an approximation for the other two cases. The strong response to curvature modulates the local burning speed. The values of approximately 0.8 and 0.5 suggest that using the laminar flame speed times the area of the flame would lead to an overestimation of the global burning by factors of approximately 1.25 and 2 , respectively. The analysis indicates that even in the flamelet regime, a Markstein correction is needed to predict the local flame speed accurately.

The way in which the burning occurs can be analyzed further by considering other joint probability density functions. Figure 11 shows the JPDFs for all five cases of temperature and carbon density, and Figure 12 shows fuel consumption rate against temperature. Here the first moment has been taken with respect to fuel consumption rate, specifically, for JPDF $P(Q, T)$ for fuel consumption rate $Q$ and temperature $T$, the first moment is defined as $Q P(Q, T)$. This highlights the burning regions and prevent the large proportion of the domain that is not burning from dominating the JPDF. Again, the PDFs have been ensembleaveraged. The solid red line in each case denotes the laminar flame correlation. In case (a), it is clear that the burning follows an almost identical path to the laminar flame. Case (b) present a similar correlation, but there is greater variability around the main burning path. In cases (c) and (d), the main burning path appears to have shifted away from the laminar flame, and there is a large amount of variability from that main path. In case (e), a dramatic change is observed, the burning path has collapsed to a single path that is significantly removed from the laminar flame. In this case, the turbulent mixing dominates the thermal diffusion, and thus the temperature and fuel cannot become decorrelated. Therefore, a single burning path is observed, which is different than the laminar flame, with very little variability.

Figure 13 shows the joint probability density function of fuel consumption rate and carbon mass fraction for case (e); again, the first moment with respect to fuel consumption rate has been taken to highlight the burning region. Similar to the corresponding temperature plot, the burning path has collapsed to a curve that is significantly different than the laminar flame, and there is little variability around it. Importantly, this figure demonstrates that the burning occurs at much lower mass fractions than in the laminar flame.

A normalized instantaneous flame structure in case (e) is compared with the laminar flame structure in Figure 14. The turbulent flame structure has been obtained by taking planar averages. Here the length scale has been normalized by the laminar flame carbon width, and each of the other quantities has been normalized by the corresponding laminar values, expect for the velocity, which has been normalized by the turbulent burning speed due to its significantly enhanced value. The turbulent structure is dramatically different. Note how the shape of the temperature and density profiles have changed, becoming closer to hyperbolic tangents. In particular, the width of the temperature profile has decreased relative to the carbon profile because the mixing is dominated by the turbulence rather than thermal diffusion. Therefore, any comparison between the turbulent and laminar flame widths will depend on the arbitrary choice of width definition. In spite of this, a rough estimate of the carbon width is approximately 60 times the laminar flame width (roughly $140 \mathrm{~cm}$ ). Some effect of thermal diffusion is still evident as the temperature profile and therefore density profile are slightly wider than the species profile; the effective Lewis number is still greater than unity. One of the biggest differences between the two flame structures is the fuel consumption rate. In the turbulent case, the fuel consumption is very much lower; the peak value is approximately an eighth of the laminar value. The physical location also appears to have shifted to a relatively higher $z$ location. It should be noted that in the distributed burning regime, the flame width is strongly affected by the integral length scale of the turbulence (and hence the domain size of the present calculations). The effect of the integral length scale will be the subject of future work but can be predicted by Damköhler scaling (Damköhler 1940), which will be discussed in $\S 4$. Some preliminary calculations (see Woosley et al. 2008; S. E. Woosley et al. 2008, in preparation) have produced encouraging results.

\section{CONCLUSIONS}

We have explored, in three dimensions, the properties of flames interacting with isotropic Kolmogorov turbulence for the conditions appropriate to a Type Ia supernova. In particular, we have examined the consequences of a turbulent energy cascade with $\varepsilon=u^{* 3} / L^{*}=10^{15} \mathrm{erg} \mathrm{g}^{-1} \mathrm{~s}^{-1}$. This might correspond, for example, to a macroscopic integral scale of $10 \mathrm{~km}$ in the supernova and speed on that scale of $100 \mathrm{~km} \mathrm{~s}^{-1}$. The range of densities explored is characteristic of the transition from the flamelet regime to distributed burning at this energy density. In particular, the Karlovitz number $\left[\left(l_{L} / l_{\mathrm{G}}\right)^{1 / 2}\right]$ varies from 0.017 at the highest density to 230 at the lowest. Two regimes of burning are clearly discernible. At the highest density, turbulence merely wrinkles an otherwise laminar flame. The requirement that the flame be resolved meant that we were not able to examine a sufficiently large integral scale $\left(l \gg l_{\mathrm{G}}\right)$ to see the multiply folded flames that should be present in the flamelet regime. The width and speed of these individual flamelets were not greatly affected by the turbulence.

As the density is decreased, the flames initially remain in the flamelet regime; however, the increase in turbulence intensity relative to the flame speed leads to enhanced wrinkling of the flame. In this regime, we see significant Lewis number effects. The combination of the sensitivity of the nuclear reaction rates to temperature and the large Lewis number leads to significant variability in the local burning rate along the flame surface, with regions of negative curvature burning much more intensely than regions of positive curvature.

For densities near $2.35 \times 10^{7} \mathrm{~g} \mathrm{~cm}^{-3}(\mathrm{Ka} \approx 3)$, turbulence begins to tear the flame, altering its width and speed, but not 

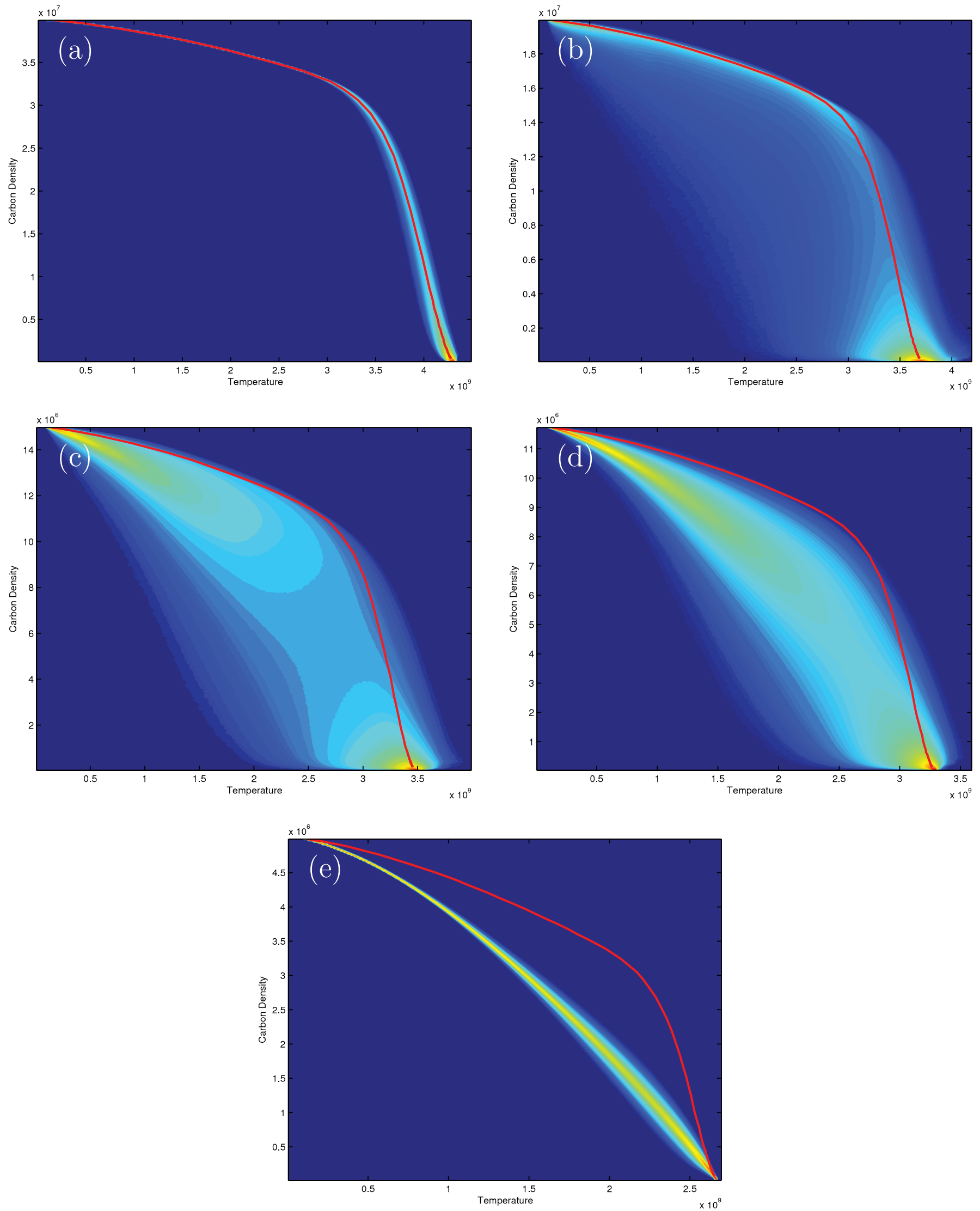

FIG. 11.- Joint probability density functions of carbon density against temperature. The red line denotes the laminar correlation. Case (a) is very close to the laminar burning path. Cases (b), (c), and (d) show greater variation around the laminar path. Case (e) present a collapse to a single curve disparate from the laminar case. This is indicative of the competition between turbulent mixing and thermal diffusion; in case (a) thermal diffusion dominates the turbulence, in case (e) turbulent mixing dominates, and in the intermediate cases the effects of both processes are of comparable importance. 

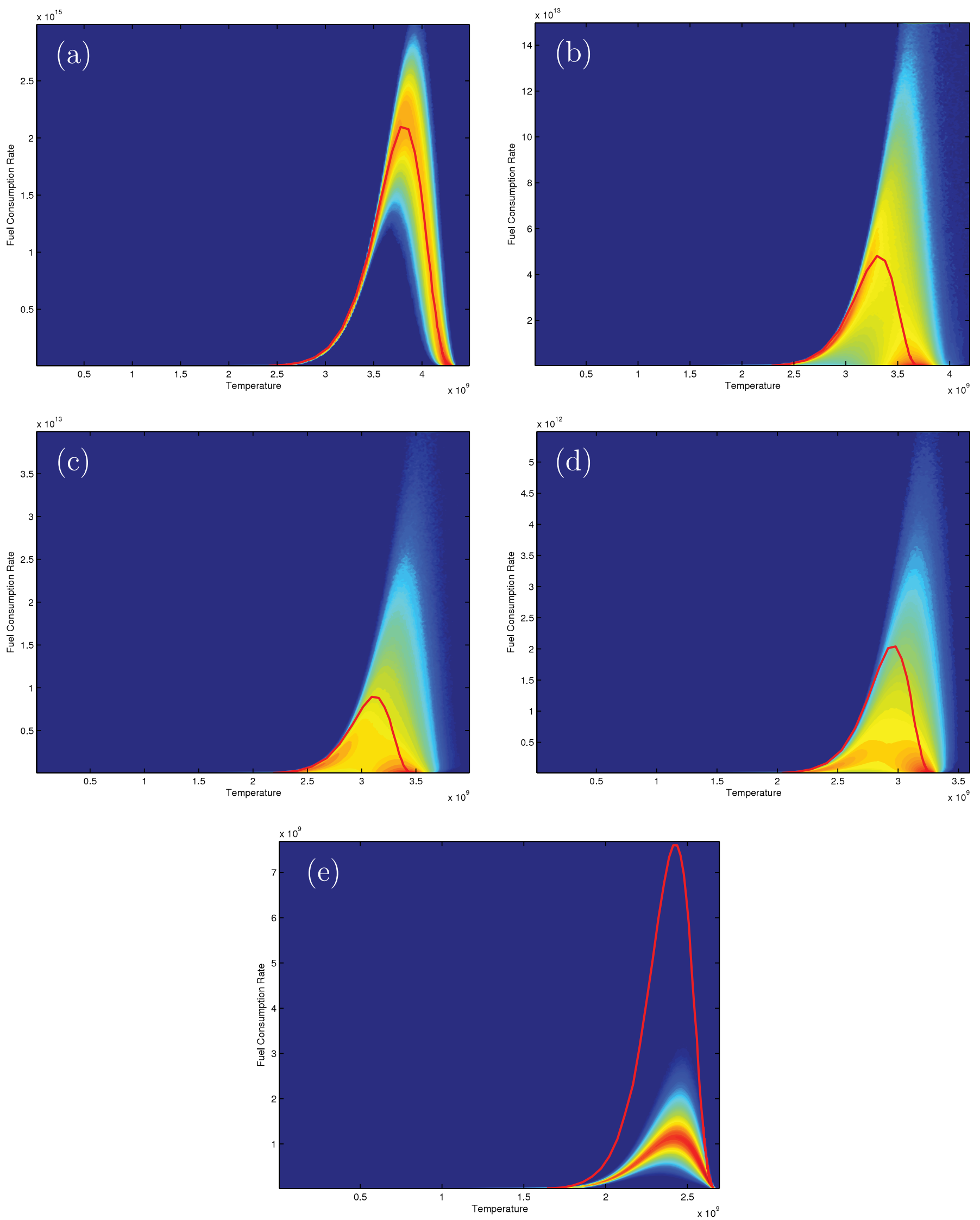

FIg. 12. - First moment with respect to fuel consumption rate of the joint probability density function of fuel consumption rate against temperature. Again, the red line denotes the laminar burning path, with which case (a) is in close agreement. Case (e) has collapsed to a curve that is different from the laminar curve and reiterates the lower local fuel consumption rate observed in this case. The intermediate cases (b) through (d) show variation around the mean path and move between the laminar and turbulent cases, and it is particularly clear that greatly increased local fuel consumption rates are observed albeit with low probability. 


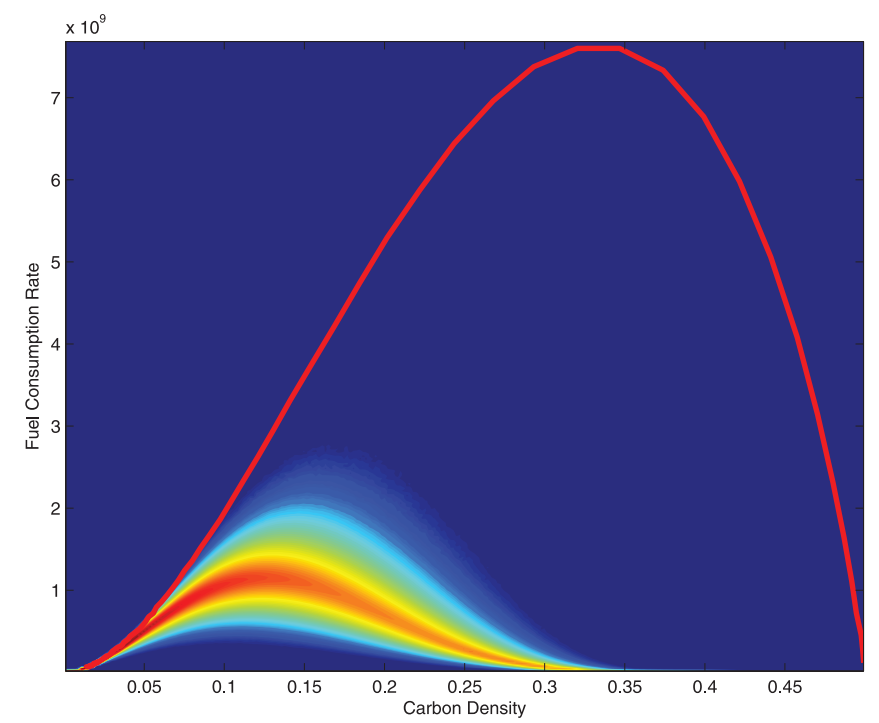

FIG. 13.-First moment with respect to fuel consumption rate of the joint probability density function of fuel consumption rate against carbon density for case (e); the other cases present similar behavior to Figs. 11 and 12. This figure again shows the collapse to a burning path different from the laminar case indicating the dominance of turbulent mixing, and the lower local fuel consumption rates in this case, but also shows that the burning occurs at lower carbon mass fractions than in the laminar case.

completely disrupting the thin region where burning is going on. This corresponds to the "thin reaction zone" regime of Peters (2000). By $1 \times 10^{7} \mathrm{~g} \mathrm{~cm}^{-3}(\mathrm{Ka}=230)$, however, the flame has been completely stirred and a qualitatively different sort of distributed burning occurs. The width of the turbulent flame is now much broader and it moves much faster. Turbulence takes over from radiation as the dominant mode of energy transport, even on small scales. Because of this, the ratio of heat diffusion to composition diffusion approaches unity; i.e., the Lewis number, which previously was very large, approaches unity. This is clearly evident in Figure 11, which shows the relation between temper- ature and fuel concentration everywhere on the grid has collapsed to a line corresponding to advective transport only.

This is the first time supernova flames have been simulated in the distributed regime in three dimensions, and our calculations may even be a first in the combustion community as well. Terrestrial flames with this degree of turbulence usually go out (Peters 2000). In a supernova, with its long timescale and large size, extinction is impossible until the star is completely disrupted. Still, our results at high Karlovitz number confirm burning in the distributed regime. Assuming Damköhler scaling (Damköhler 1940), the turbulent flame speed $s_{T}$ and its width $l_{T}$ should obey the scaling relations

$$
\begin{gathered}
s_{T}=\sqrt{\frac{D_{T}}{\tau_{\text {nuc }}^{T}}}, \\
l_{T}=\sqrt{D_{T} \tau_{\text {nuc }}^{T}},
\end{gathered}
$$

for a turbulent diffusion coefficient $D_{T} \sim u^{\prime} l$ and nuclear timescale $\tau_{\text {nuc }}^{T}$. It follows that

$$
\frac{s_{T}}{s_{L}}=\left(\frac{u^{\prime} l}{s_{L} l_{L}}\right)^{1 / 2}\left(\frac{\tau_{\mathrm{nuc}}^{L}}{\tau_{\mathrm{nuc}}^{T}}\right)^{1 / 2},
$$

where $\tau_{\text {nuc }}^{L}$ is the laminar nuclear timescale. A key point is that the nuclear timescale is different in the laminar and distributed cases. Because of the different distributions of temperature and carbon abundance in the two cases (Fig. 11), the nuclear timescale is almost an order of magnitude longer in the turbulent case. From the information in Table 1 , for case (e), $\tau_{\text {nuc }}^{L}=l_{L} / s_{L}=$ $6.5 \times 10^{-4} \mathrm{~s}$, while for the turbulent flame (Fig. 14), $\tau_{\text {nuc }}^{T}=$ $l_{T} / s_{T}=7.2 \times 10^{-3} \mathrm{~s}$. This gives $s_{T} / s_{L} \approx 6.4$, approximately $15 \%-20 \%$ higher than what was calculated (Fig. 7). The overestimate is reasonable since the average turbulent diffusion coefficient is likely to be somewhat less than that derived for the largest possible length scale, i.e., $D_{T}<u^{\prime} l$.
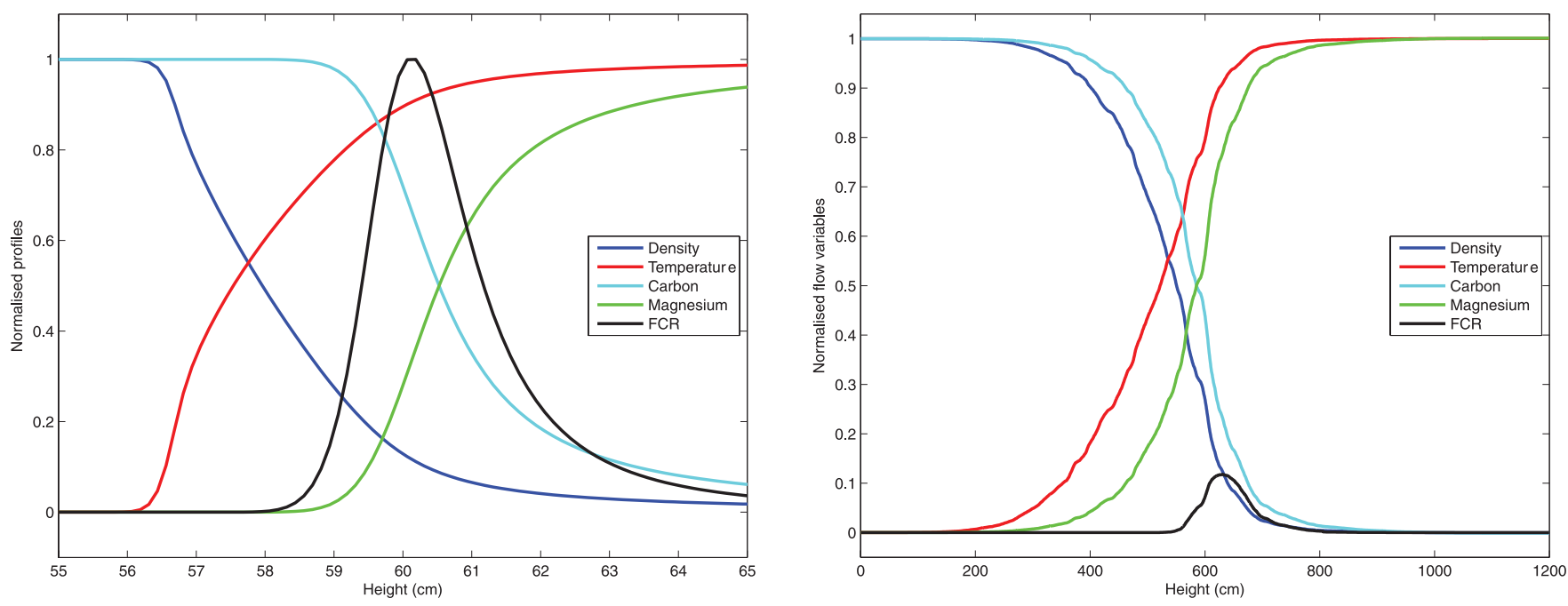

FIG. 14. - Comparison of laminar (left) and turbulent (right) profiles in case (e). In particular, note the disparate length scales between the two figures; the turbulent

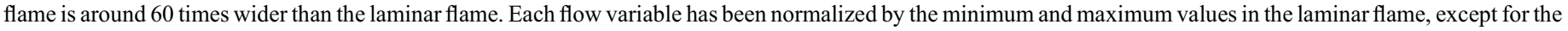

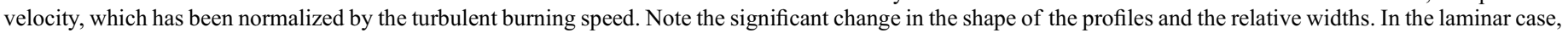

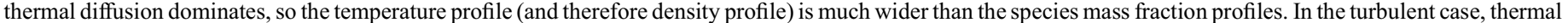

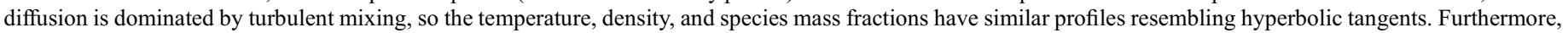
the fuel consumption rate is greatly reduced in the turbulent case, but because of the greatly broadened flame width, the overall fuel consumption rate is enhanced. 
In the distributed burning regime with Karlovitz number greater than 10 and Damköhler number less than 1, i.e., case (e), the mixing is dominated by turbulence and has a shorter timescale than the chemical timescale. Therefore, the overall reaction rate is limited by the chemistry that can occur along the burning path prescribed by Figure 11e, so the turbulent nuclear timescale is a constant. Together with the calculations presented in Woosley et al. (2008; S. E. Woosley et al. 2008, in preparation) we believe that case (e) has achieved the asymptotic value of $\tau_{\text {nuc }}^{T}$.

For larger integral scales than those simulated here, assuming that $\tau_{\text {nuc }}^{T}$ remains fixed, the turbulent speed $s_{T}$ and flame width $l_{T}$ would both increase as $l^{2 / 3}$, as can be seen from equation (7) and the fact that $u^{\prime} \propto l^{1 / 3}$. Such scaling cannot continue indefinitely, however, without eventually encountering the limit $s_{T} \lesssim u^{\prime}$. The flame cannot move much faster than the turbulence that carries it.

For our assumed turbulent energy and composition, the length scale where $l^{2 / 3}$ scaling breaks down is predicted to be

$$
l_{\lambda}=\left(\frac{u^{\prime}}{s_{T}}\right)^{3} l
$$

or, for $s_{T}=1.95 \times 10^{4} \mathrm{~cm} \mathrm{~s}^{-1}$ at $u^{\prime}=2.47 \times 10^{5} \mathrm{~cm} \mathrm{~s}^{-1}$ and $l=15 \mathrm{~cm}$, as calculated here, $l_{\lambda} \sim 300 \mathrm{~m}$. This compares reasonably well with the 155 m estimated by Woosley (2007) using a very simplified representation of the flame structure.

The actual integral scale in the supernova is much larger even than this large value. There, one expects a different sort of burning reflecting some distribution of turbulently broadened flame- lets with characteristic scale $l_{\lambda}$ embedded in an overall flame brush as large as the actual integral scale, $L^{*} \sim 10 \mathrm{~km}$. Exploratory calculations in progress suggest a highly variable flame width and speed in that domain that may be conducive to spontaneous detonation.

As a practical consequence, the domination of turbulent transport at low density means one no longer needs to resolve the laminar flame scale. It thus becomes possible to carry out meaningful simulations using a much larger grid. In our next paper, we will demonstrate the validity of a simple subgrid model for the turbulent transport and carry out three-dimensional calculations that take us into the unsteady burning regime.

The authors thank F. X. Timmes for making his equation of state and conductivity routines available online. Support for A. J. A. was provided by a Seaborg Fellowship at Lawrence Berkeley National Laboratory under contract DE-AC02-05CH11231. Support for J. B. B. and M. S. D. was provided by the SciDAC Program of the Office of Advanced Scientific Computing Resarch of the U.S Department of Energy under contract DE-AC0205CH11231. At UCSC this research has been supported by the NASA Theory Program NNG05GG08G and the DOE SciDAC Program (DE-FC02-06ER41438). M. Z. was supported by a DOE/Office of Nuclear Physics Outstanding Junior Investigator award, grant DE-FG02-06ER41448 to SUNY Stony Brook. The computations presented here were performed on the Jaguar XT4 at ORNL as part of an INCITE award and on the ATLAS Linux Cluster at LLNL as part of a Grand Challenge Project.

\section{REFERENCES}

Aspden, A. J., Nikiforakis, N., Dalziel, S. B., \& Bell, J. B. 2008, Characterizing Implicit LES Methods (Berkeley: CCSE), submitted

Bell, J. B., Day, M. S., Grcar, J. F., \& Lijewski, M. J. 2006, Commun. Appl. Math. Comput. Science, 1, 29

Bell, J. B., Day, M. S., Rendleman, C. A., Woosley, S. E., \& Zingale, M. A. 2004a, J. Comput. Phys., 195, 677 2004b, ApJ, 608, 883

Boris, J. P., Grinstein, F. F., Oran, E. S., \& Kolbe, R. L. 1992, Fluid Dyn. Res., 10,199

Cabot, W. H., \& Cook, A. W. 2006, Nature Phys., 2, 562

Chertkov, M. 2003, Phys. Rev. Lett., 91, 115001

Damköhler, G. 1940, Z. Elektrochem, 46, 601

Day, M., Bell, J., Bremer, P.-T., Pascucci, V., Beckner, V., \& Lijewski, M. 2008, Combustion Flame, in press

Dursi, L. J., \& Timmes, F. X. 2006, ApJ, 641, 1071

Gamezo, V. N., Khokhlov, A. M., \& Oran, E. S. 2005, ApJ, 623, 337

Gamezo, V. N., Khokhlov, A. M., Oran, E. S., Chtchelkanova, A. Y., \& Rosenberg, R. O. 2003, Science, 299, 77

Garland, M. 1999, Ph.D. thesis, Carnegie Mellon Univ.

Golombek, I., \& Niemeyer, J. C. 2005, A\&A, 438, 611

Grinstein, F. F., \& Fureby, C. 2004, Comput. Sci. Eng., 6, 37

Grinstein, F. F., Margolin, L. G., \& Rider, W. J. 2007, Implicit Large Eddy Simulation (Cambridge: Cambridge Univ. Press)

Khokhlov, A. M. 1991, A\&A, 245, 114

Khokhlov, A. M., Oran, E. S., \& Wheeler, J. C. 1997, ApJ, 478, 678

Lisewski, A. M., Hillebrandt, W., \& Woosley, S. E. 2000a, ApJ, 538, 831

Lisewski, A. M., Hillebrandt, W., Woosley, S. E., Niemeyer, J. C., \& Kerstein, A. R. 2000b, ApJ, 537, 405
Margolin, L. G., Rider, W. J., \& Grinstein, F. F. 2006, J. Turbulence, 7, 1

Niemeyer, J. C. 1999, ApJ, 523, L57

Niemeyer, J. C., \& Hillebrandt, W. 1995, ApJ, 452, 769

Niemeyer, J. C., \& Kerstein, A. R. 1997, NewA, 2, 239

Niemeyer, J. C., \& Woosley, S. E. 1997, ApJ, 475, 740

Pan, L., Wheeler, J. C., \& Scalo, J. 2008, ApJ, 681, 470

Peters, N. 2000, Turbulent Combustion (Cambridge: Cambridge Univ. Press)

Porter, D. H., Pouquet, A., \& Woodward, P. R. 1992, Phys. Rev. Lett., 68, 3156

Röpke, F. K. 2007, ApJ, 668, 1103

Röpke, F. K., \& Hillebrandt, W. 2005, A\&A, 431, 635

Röpke, F. K., Hillebrandt, W., \& Niemeyer, J. C. 2004, A\&A, 421, 783

Sharp, D. H. 1984, Physica, 12D, 3

Timmes, F. X. 2000, ApJ, 528, 913

Timmes, F. X., \& Swesty, F. D. 2000, ApJS, 126, 501

Timmes, F. X., \& Woosley, S. E. 1992, ApJ, 396, 649

Weaver, T. A., Zimmerman, G. B., \& Woosley, S. E. 1978, ApJ, 225, 1021

Woosley, S. E. 2007, ApJ, 668, 1109

Woosley, S. E., Aspden, A. J., Bell, J. B., Day, M. S., Kerstein, A. R., \& Sankaran, V. 2008, in J. Phys. Conf. Ser. 125, SciDAC 2008, ed. R. Stevens (Bristol: IOP), 012012

Youngs, D. L. 1991, Phys. Fluids A, 4, 1312

Zingale, M., Woosley, S. E., Bell, J. B., Day, M. S., \& Rendleman, C. A. 2005a, in J. Phys. Conf. Ser. 16, SciDAC 2005, ed. A. Mezzacappa (Bristol: IOP), 405 Zingale, M., Woosley, S. E., Rendleman, C. A., Day, M. S., \& Bell, J. B. 2005b, ApJ, 632, 1021 